
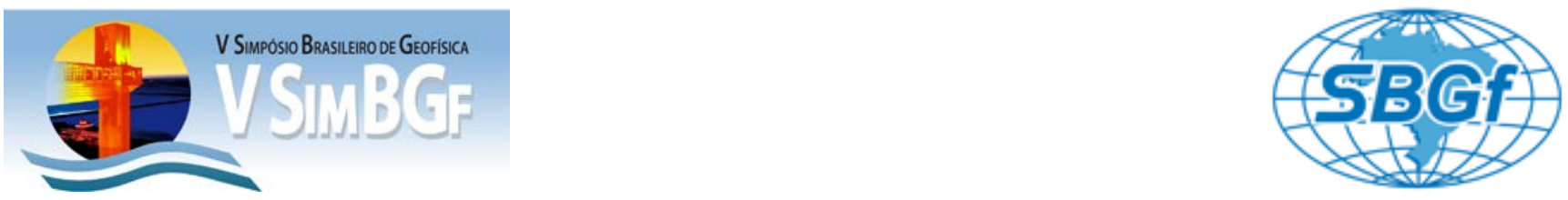

\title{
Análise das observações do gravímetro digital Scintrex CG5 na linha de calibração vertical Observatório Nacional - Agulhas Negras
}

Francismar Rimoli Berquó*, Observatório Nacional/MCTI, Instituto Federal Fluminense/campus Itaperuna, Brasil. Íris Pereira Escobar, Uerj, Brasil.

Andrés R.R. Papa, Uerj e Observatório Nacional/MCTI, Brasil.

Alcides Antônio dos Santos, Observatório Nacional/MCTI, Brasil.

Copyright 2012, SBGf - Sociedade Brasileira de Geofísica

Este texto foi preparado para a apresentação no $V$ Simpósio Brasileiro de Geofísica, Salvador, 27 a 29 de novembro de 2012. Seu conteúdo foi revisado pelo Comitế Técnico do V SimBGf, mas não necessariamente representa a opinião da SBGf ou de seus associados. É proibida a reprodução total ou parcial deste material para propósitos comerciais sem prévia autorização da SBGf.

\section{Abstract}

O presente trabalho tem o principal objetivo de verificar o comportamento instrumental utilizando os mais modernos gravímetros diferenciais Scintrex CG5 na Linha de Calibração Observatório Nacional - Agulhas Negras. A verificação do ensaio de calibração desses instrumentos diferenciais foi realizada pelo método dos mínimos quadrados com o modelo matemático linear desenvolvido por Dias e Escobar (2001). São apresentados os resultados obtidos na aferição de 3 gravímetros diferenciais Scintrex CG5. O gravímetro Scintrex CG5 possui uma resolução das leituras instrumentais de $1 \mu \mathrm{Gal}$. Valores de resíduos na ordem do microgal são esperados após o ajustamento. O maior valor residual após o ajustamento para as leituras instrumentais foi de $6,5.10^{-3} \mathrm{UI}$, que ficaram abaixo do desvio padrão estimado inicialmente para essas grandezas observáveis $\left(1.10^{-2} \mathrm{UI}\right)$.

\section{Introdução}

A gravimetria tem experimentado desenvolvimento considerável nos últimos anos, tanto na técnica de medição absoluta como na relativa. $O$ avanço na gravimetria relativa foi 0 desenvolvimento dos gravímetros digitais Scintrex modelo CG-5 (CG5) que possuem um sistema eletrônico que veio substituir os dispositivos analógicos, tendo algumas vantagens, por exemplo, as observações são medidas automaticamente e registradas digitalmente com as informações sugeridas no levantamento, possui uma função de calibração mais simples comparado com os gravímetros LaCoste\&Romberg (LCR) e suas observações não possuem erros periódicos. Esses instrumentos possibilitam observações com precisão de alguns microgals $\left(5 \mu \mathrm{Gal}=50 \mathrm{~nm} / \mathrm{s}^{2}\right)$, o que isso significa um aprimoramento de pelo menos uma ordem de grandeza em relação aos instrumentos anteriores, por exemplo, os LCR.

A natural instabilidade das características do sistema elástico torna necessário submeter os gravímetros diferenciais digitais CG5, a avaliações e aferições em pontos onde a gravidade seja conhecida (Oja et al, 2010). Assim, é possível controlar eventuais variações na escala, bem como verificar o bom funcionamento dos dispositivos que evitam ou compensam os efeitos de variações das condições ambientais nas leituras do gravímetros, notadamente os efeitos relacionados com a variação da temperatura e de pressão atmosférica. Segundo Escobar et al(1996), um dos métodos usados para aferição dos gravímetros diferenciais é a observação periódica de suas leituras em trajetos entre pontos de gravidade conhecida, denominados linhas de calibração.

A Linha de Calibração vertical Observatório Nacional Agulhas Negras (LCONAN) é composta por 5 estações gravimétricas da Rede Gravimétrica Fundamental Brasileira (RGFB) (Escobar, 1987) com uma amplitude de $628 \mathrm{mGal}$ desmembradas em quatro intervalos de gravidade (Fig.1 e Tab.1). Neste trabalho, foram utilizados 3 CG5, onde 2 pertencem ao $\mathrm{PegBr}$, com $\mathrm{n}^{\text {os }}$ de série 40343 e 40347 (Pool de Equipamentos Geofísicos do Brasil) e 1, ao Departamento de Geofísica do Observatório Nacional com $\mathrm{n}^{\circ}$ de série 40600. A verificação da calibração desses instrumentos diferenciais foi realizada pelo método dos mínimos quadrados (MMQ) com o modelo matemático linear desenvolvido por Dias e Escobar (2001) para ajustamento gravimétrico, aqui denominado modelo D\&E. Este modelo utiliza grandezas observáveis as leituras instrumentais corrigidas da deriva e maré expressas em unidades de leituras e os coeficientes das funções de calibração dos gravímetros e como incógnitas os valores de gravidade e os fatores lineares de escala.

\section{A Linha de Calibração Vertical Observatório Nacional - Agulhas Negras}

Segundo Escobar et al (1996), o IAG/USP teve a iniciativa da implantação dessa linha de calibração gravimétrica vertical até o Parque Nacional de Itatiaia, localizado no posto do Ibama, com objetivo de monitorar os gravímetros diferenciais, aqui nomeada de LCONAN. Essa linha de calibração explora a variação da gravidade com a altitude e também o comportamento desses instrumentos com a variação da temperatura e pressão podendo verificar o bom desempenho dos dispositivos térmicos e barométricos.

O percurso do Observatório Nacional até o Parque de Itatiaia tem aproximadamente 250 quilômetros de distância e envolve uma diferença de altitude da ordem de 2500 metros e uma significativa variação de temperatura e pressão. O levantamento gravimétrico dos gravímetros CG5 totalizou 72 observações (Tab.2). 


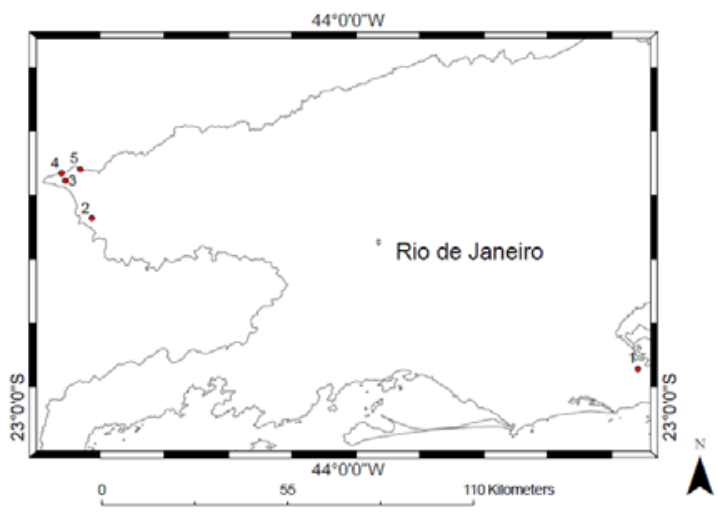

Figura 1 - A distribuição das 5 estações da LCONAN: 1 016080; 2- CAL02; 3- CAL03; 4- CALO4; 5- CAL05.

Tabela 1 As estações gravimétricas, da LCONAN, e os valores dos intervalos de gravidade.

\begin{tabular}{|c|c|c|c|}
\hline $\begin{array}{l}\text { Código da } \\
\text { Estação }\end{array}$ & Latitude & Longitude & Altitude \\
\hline 016080 & $-22^{\circ} 53.4^{\prime}$ & $-43^{\circ} 13.4^{\prime}$ & $18 \mathrm{~m}$ \\
\hline CAL02 & $-23^{\circ} 29.8^{\prime}$ & $-44^{\circ} 19.1^{\prime}$ & $500 \mathrm{~m}$ \\
\hline CAL03 & $-22^{\circ} 24.2^{\prime}$ & $-44^{\circ} 45.1^{\prime}$ & $1300 \mathrm{~m}$ \\
\hline CAL04 & $-22^{\circ} 22.6^{\prime}$ & $-44^{\circ} 45.6^{\prime}$ & $1669 \mathrm{~m}$ \\
\hline CAL05 & $-22^{\circ} 22.4^{\prime}$ & $-44^{\circ} 42.4^{\prime}$ & $2446 \mathrm{~m}$ \\
\hline \multicolumn{4}{|c|}{ Intervalos de gravidade } \\
\hline \multicolumn{3}{|c|}{$2016080 \rightarrow$ CAL02 } & $191 \mathrm{mGal}$ \\
\hline \multicolumn{3}{|c|}{ CAL02 $\rightarrow$ CAL03 } & $181 \mathrm{mGal}$ \\
\hline \multicolumn{3}{|c|}{ CAL03 $\rightarrow$ CAL04 } & $94 \mathrm{mGal}$ \\
\hline \multicolumn{3}{|c|}{ CAL04 $\rightarrow$ CAL05 } & $162 \mathrm{mGal}$ \\
\hline
\end{tabular}

Tabela 2 Estatística das observações dos gravímetros CG5 utilizados na LCONAN.

\begin{tabular}{c||c|c|c|c|c|c}
\hline \multirow{2}{*}{ CG5 } & \multicolumn{2}{|c|}{ Leituras (UI } & \multicolumn{4}{c}{ Intervalo de gravidade } \\
\cline { 2 - 7 } & Menor & Maior & Bons & $\%$ & Rejeitados & $\%$ \\
\hline \hline 40343 & 7403. & 8027. & 24 & 100 & 0 & 0 \\
\hline 40347 & 3290. & 3911. & 24 & 100 & 0 & 0 \\
\hline 40600 & 2812. & 3433. & 24 & 100 & 0 & 0 \\
\hline \hline
\end{tabular}

* Unidades Instrumentais

\section{3 - As observações oriundas dos gravímetros digitais Scintrex CG5}

O gravímetro CG5 é baseado em um sistema elástico de quartzo fundido cobrindo uma amplitude de gravidade de aproximadamente $8000 \mathrm{mGal}$ sem sofrer ajuste, tendo uma resolução de leitura de $1 \mu \mathrm{Gal}$. Dependendo dos parâmetros iniciais selecionados pelo operador, as correções instrumentais e da maré luni-solar podem ser calculadas e aplicadas para cada observação durante a aquisição dos dados automaticamente (Fig.2b). Neste trabalho, essas correções foram selecionadas e aplicadas pelo sistema inerente no instrumento nas observações.

O sistema de sensibilidade do gravímetro é selado numa câmera de vácuo para protegê-lo das variações da pressão atmosférica e da temperatura ambiental. Sendo baseado em uma mola de quartzo fundido, o CG-5 é insensível a fortes variações magnéticas. Pequenas variações da temperatura ambiental são estimadas e as observações são corrigidas desse efeito. O CG5 possui um filtro sísmico que elimina ruídos nas observações. $O$ gravímetro tem um nível eletrônico que é nivelado através de três calantes onde os sentidos das rotações são indicados na parte superior da tela de operação (Fig.2a). Além disso, o gravímetro calcula as correções luni-solares, baseado nas expressões de Longman (Longman, 1959), utilizando um fator gravimétrico de 1,16 (Freitas, 1993).

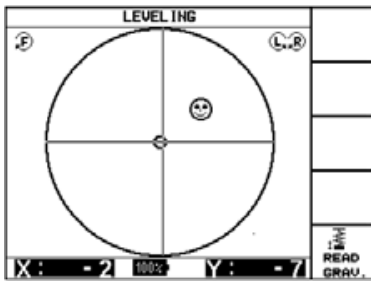

(a)

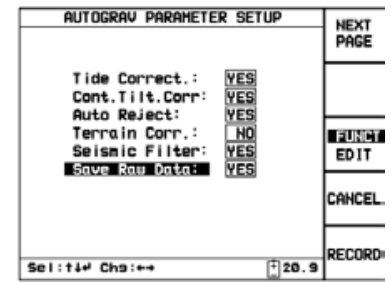

(b) (Scintrex.2010)
Figura 2 (a) A tela de nivelamento do CG5 quando ele está nivelado; (b) as correções que o sistema eletrônico do CG5 aplicou automaticamente nas observações medidas na LCONAN.

Para iniciar um trabalho geofísico, com o uso do gravímetro CG5, é obrigatoriamente necessário o uso do tripé. O sensor do gravímetro fica numa altura específica da estação gravimétrica durante as medidas $(\Delta h)$ (Fig.3) (Scintrex, 2010, p.1-8). Então, precisa-se aplicar a correção dessa elevação $(\Delta \zeta)$ que é dada por:

$$
\Delta \zeta=\text { Gradiente } x \Delta h
$$

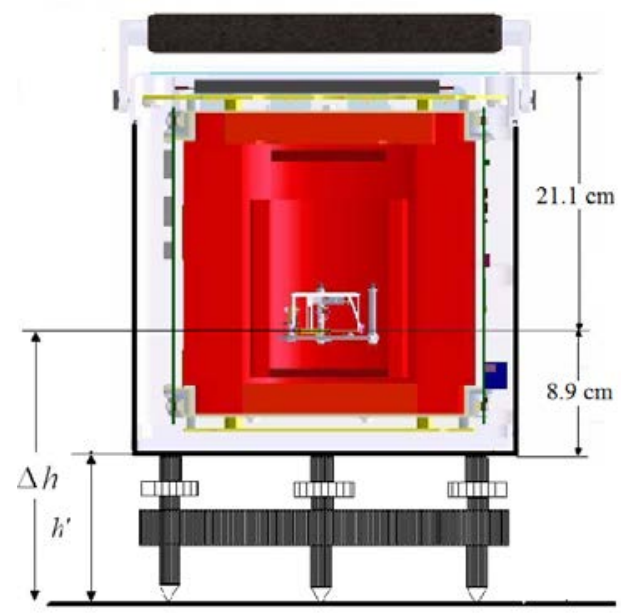

Figura 3 Esquema do gravímetro CG5.

O valor da altura $h$ ' é medido pelo operador antes de iniciar as medidas, em $\mathrm{cm}$. Os valores dos gradientes nas estações da LCONNA foram medidos por Sousa e Santos (2010), exceto da estação 016080 que foi medido com os gravímetros CG5, e usados nesse trabalho (Fig.4).

Existem alguns parâmetros instrumentais que devem ser periodicamente verificados e se possível ajustados, dentre eles temos a deriva instrumental que foi corrigida 
para cada trecho da LCONAN. Este parâmetros foi corrigido na análise prévia das observações antes do ajustamento.

Tratando-se de uma campanha tendo por objetivo de verificar o comportamento dos gravimetros CG5, números de série 40343, 40347 e 40600, adotaram-se procedimentos de campo adequados a levantamentos de precisão. Os circuitos terrestres de ida-e-volta, do tipo A$\mathrm{B}-. . . \mathrm{-N}-. . .-\mathrm{B}-\mathrm{A}$, foram desenvolvidos para controlar a deriva instrumental de modo eficientes e também eventuais erros nas observações individuais e saltos (variações bruscas e intensas das leituras).

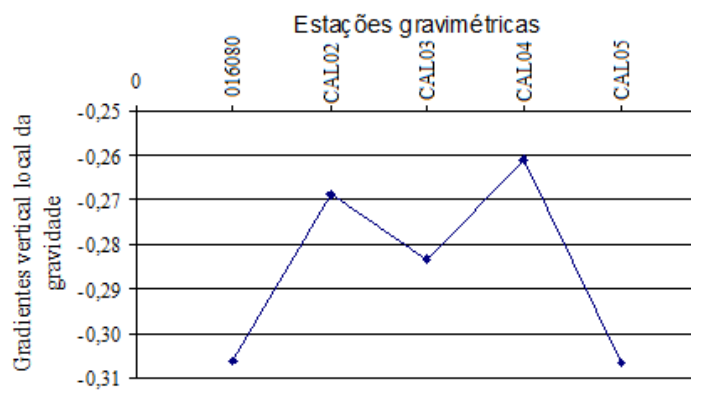

Figura 4 Valores dos gradientes verticais locais da gravidade nas estações da LCONAN.

Neste trabalho, essas observações foram medidas com os gravímetros CG5 sobre o local a ser observado um de cada vez. Cada percurso de ida-e-volta foi concluído num intervalo máximo de 14 horas. Agrupando as observações referentes aos mesmos intervalos de gravidade por gravímetro que participaram na aquisição das leituras instrumentais, tivemos 12 intervalos de gravidade distintos que foram inseridos no ajustamento da LCONAN. O número de leituras determinado, nos locais, foi 3 com um intervalo de tempo de 1 minuto entre elas. Antes de iniciar as leituras instrumentais, o gravímetro CG5 ficou alocado e nivelado sobre o local a ser observado durante 20 minutos para o acomodamento do sistema elástico. Sabendo das possíveis vibrações que podem ocorrer durante o transporte até o local a ser medido, todas as observações sofreram correções de deriva dinâmica antes do ajustamento (Yushkin, 2011).

Inicialmente, esses dados passaram por duas avaliações prévias para verificar a qualidade de todos os intervalos de gravidade. Esses procedimentos foram adotados para determinar os valores das leituras, corrigidas de maré, de elevação e de deriva instrumental, em UI, para os pares de estações gravimétricas nos intervalos de gravidade. A determinação da correção do efeito da maré luni-solar $\left(\xi_{o}\right)$, em UI, foi determinado da seguinte forma.

$$
\xi=\frac{\xi_{o}}{\alpha},
$$

onde: $\xi$ é o valor da correção da maré luni-solar, em UI; $\xi_{o}$ é o valor da correção da maré luni-solar, em mGal, estimado pelo gravímetro CG5; $\alpha$ é o coeficiente da função de calibração dada pela tabela do gravímetro referente ao valor da leitura instrumental.

Os intervalos de gravidade foram estimados pela média aritmética ponderada de acordo com os inversos dos intervalos de tempo. Assim, tem-se:

$$
\bar{\Delta} l_{i j}=\frac{\sum_{z=1} \frac{\left|\Delta l_{z}\right|}{\Delta t_{z}}}{\sum_{z=1} \frac{1}{\Delta t_{z}}}
$$

Como critério de rejeição para os intervalos de gravidade observados adotou-se o seguinte:

$$
\begin{gathered}
\left|\Delta l_{i j}-\bar{\Delta} l_{i j}\right| \leq 0,05 U I \\
\Delta t_{i j} \leq 24 \text { horas }
\end{gathered}
$$

Se algum intervalo de gravidade não satisfazer as Eqs. (4) e (5), o intervalo de gravidade é rejeitado e uma nova média é calculada para o mesmo par de estações gravimétricas até que todas as observações satisfaçam estas equações.

\section{O modelo matemático de D\&E}

Muitos trabalhos de calibração de gravímetros diferenciais utilizam-se polinômios (Faggion et al, 2002). Dias e Escobar (2001), abandonou a idéia de interpolação polinomial e desenvolveu um modelo matemático linear que segue a idéia do próprio fabricante dos gravímetro LCR, onde eles utilizam os procedimentos de conversão das leituras instrumentais em UI para mGal no próprio modelo matemático. Com isso, os coeficientes das funções de calibração são objetos de estudo.

As aferições dos gravímetros diferenciais CG5 foram realizadas pelo MMQ com o modelo D\&E (Dias e Escobar, 2001), que é um modelo linear e aplicado para qualquer gravímetro diferencial, onde envolve os coeficientes das funções de calibração dos gravímetros $(\alpha)$, as leituras instrumentais $(l)$, os valores de gravidade $(g)$ e os fatores lineares de escala $\left(k_{r}\right)$ dos gravímetros $(r)$.

$$
\begin{aligned}
g_{j}-g_{i}+k_{r}\left(l_{i r} \alpha_{I}-l_{j r} \alpha_{J}-\delta I_{r} \alpha_{I}+\right. \\
\left.\delta J_{r} \alpha_{J}+\delta \sum_{m=0}^{I-1}{ }_{r} \alpha_{m}-\delta \sum_{m=0}^{J-1}{ }_{r} \alpha_{m}\right)=0
\end{aligned}
$$

onde: $g$ são os valores de gravidade; $l$ são os valores das leituras; $(\alpha)$ são os coeficientes das funções de calibração; $k_{r}$ são os fatores lineares de escala para cada gravímetro $(r) ; I$ é igual ao número inteiro de intervalos discretizados contidos em $I_{i}\left(I=\mathfrak{J}\left(\frac{l_{i}}{100}\right)\right), J$ é análogo a $I$ para a leitura $l_{j ;} \quad \delta$ é a discretização por intervalo para o coeficiente igual a 100. 
Como grandezas observáveis são utilizadas as leituras instrumentais, corrigidas em UI, e os coeficientes das funções de calibração dos gravímetros usados nas medições $(\alpha)$. Os parâmetros incógnitos a serem determinados em ajustamento pelo MMQ são os valores de gravidade nas estações da LCONAN e os fatores lineares de escala dos gravímetros $\left(k_{r}\right)$. A função de calibração do gravímetro CG5 é linear em todo o alcance da escala, portanto a conversão de UI para mGal é feita por um único fator de escala, determinado pelo fabricante, e automaticamente corrigido pelo sistema residente no instrumento. Assim, as leituras fornecidas pelo instrumento já são expressas em mGal. Entretanto, para efeito de aferição do instrumento, é importante verificar a coerência deste com o datum gravimétrico local. Essa verificação foi feita utilizando-se o modelo D\&E, para cuja aplicação foi construída a tabela 3, onde a amplitude de escala é discretizada em intervalos de $100 \mathrm{UI}$, para os quais os fatores de conversão mGal/UI iniciais foram igualados à unidade.

Tabela 3 Parte da tabela construída para os gravímetros CG5.

\begin{tabular}{c||c||c}
\hline \hline \multicolumn{2}{c|}{ Leituras instrumentais } & $\begin{array}{c}\text { Coeficientes para o } \\
\text { intervalo }(\alpha)\end{array}$ \\
\hline \hline em UI & em mGal & 1.00000 \\
\hline 0 & 0 & 1.00000 \\
\hline 100 & 100 & 1.00000 \\
\hline 200 & 200 & $\ldots$ \\
\hline$\ldots$ & $\ldots$ & $\ldots$ \\
\hline \hline
\end{tabular}

A solução para esse modelo matemático requer no mínimo dois valores de referência. Recentemente, o Observatório Nacional adquiriu um gravímetro absoluto Micro-g LaCoste A-10\#011 (Fig.5), aqui nomeado de A-10, que foi utilizado nas estações contidas na tabela 1 (Tab.4) (Sousa e Santos, 2010). Como toda medida experimental possui erros de diversas fontes, os valores da tabela 4 foram introduzidos como injunções relativas com um modelo adicional (Escobar, p.56, 1986):

$$
g-\bar{g}=0
$$

onde $g$ são os valores de gravidade absolutos observados nas estações de referência e $g$ é o mesmo na Eq.(6).

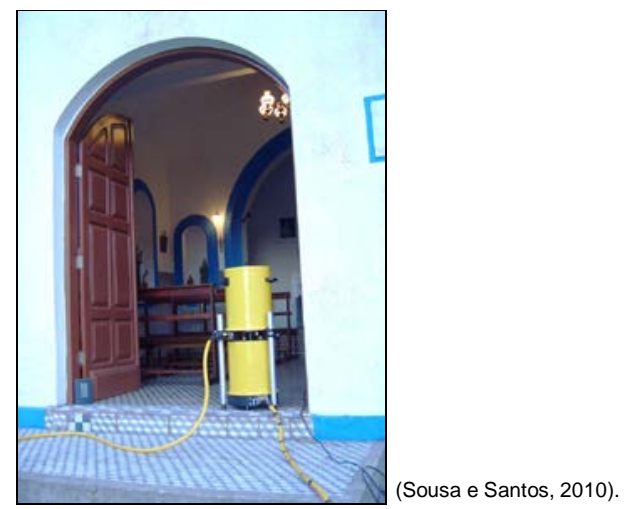

Figura 5 o gravímetro A-10 em operação na estação CAL03.
Tabela 4 Os valores absolutos da gravidade nas estações gravimétricas da LCONAN.

\begin{tabular}{c|c|c}
\hline $\begin{array}{c}\text { Código das } \\
\text { estações }\end{array}$ & $\begin{array}{c}\mathrm{g} \\
(\mu \mathrm{Gal})\end{array}$ & $\begin{array}{c}\sigma \\
(\mu \mathrm{Gal})\end{array}$ \\
\hline \hline $016080^{1}$ & 978791665 & 8 \\
\hline CAL02 & 978601078 & 6 \\
\hline CAL03 & 978419483 & 9 \\
\hline CAL04 & 978325514 & 8 \\
\hline CAL05 & 978163000 & 8 \\
\hline \hline
\end{tabular}

\section{Resultados e Discussões}

O método combinado foi utilizado para ajustar a LCONAN. A matriz dos pesos usada no ajustamento teve diferentes precisões: (i) para as leituras instrumentais os pesos foram iguais ao inverso de suas variâncias separadamente para cada gravímetro CG5 com um desvio padrão de $1.10^{-2} \mathrm{UI}$, estimado pelo erro quadrático médio; (ii) Não existe uma indicação explícita da precisão para os coeficientes da função de calibração dos gravímetros. Portanto, foi truncado um desvio padrão de $1.10^{-5} \mathrm{mGal} / \mathrm{Ul}$ e a ponderação com o inverso da variância; (iii) os pesos dos valores de gravidade de referência são inversos de suas variâncias (Tab.4).

A contribuição de cada observação, $l_{i}$, à redundância $n$ recebe o nome de redundância parcial (Santos Júnior et al, 2005). As observações do CG5 tiveram 12 observações pertencentes ao intervalo referente à suficiente controlabilidade e as outras $12 \mathrm{com}$ boa controlabilidade. Indicando que as observações do CG5 têm, no mínimo, uma controlabilidade suficiente na presença de erros grosseiros.

A variância a priori $\left(\sigma_{o}^{2}\right)$ da unidade de peso foi igual a 1. Resolvendo a Eq.6 pelo MMQ, a estimativa da variância a posteriori será dada por (Gemael, 1994):

$$
\hat{\sigma}_{o}^{2}=\frac{V^{T} P V}{n}
$$

onde $n$ é o grau de liberdade; $V$ é o vetor dos resíduos; $P$ é a matriz de pesos para as observações.

O valor da variância a posteriori foi 1,10 , que a partir do teste global com nível de significância de 5\% ficou dentro do intervalo, ou seja, a hipótese básica não é rejeitada $\left(\sigma_{o}^{2}=\hat{\sigma}_{o}^{2}\right)$ (Tab.5) (Gemael, 1994).

Os valores dos resíduos estimados para os valores absolutos de gravidade ficaram abaixo de dois desvios padrão estimado inicialmente no ajustamento (Tab.6), mostrando uma qualidade boa das observações medidas com os gravímetros CG5.

\footnotetext{
1 O valor da gravidade nesta estação gravimétrica foi transportado a partir da estação absoluta 010176 medida com o gravímetro absoluto Micro-g LaCoste A-10\#011.
} 
Tabela 5 Teste Global com nível de significância de 5\% e Teste Data Snooping.

\begin{tabular}{l|c}
\multicolumn{1}{c|}{ Critérios utilizados } & $\begin{array}{c}\text { Valores do } \\
\text { ajustamento }\end{array}$ \\
\hline \hline Intervalos distintos da LCONAN & 12,0 \\
\hline Grau de liberdade da LCONAN & 9,00 \\
\hline Variância a priori & 1,00 \\
\hline Variância a posteriori & 1,10 \\
\hline Qui-quadrado teórico & {$[2,70 ; 19,02]$} \\
\hline Qui-quadrado calculado & 9,90 \\
\hline Observações localizadas com erro & Nenhuma \\
\hline \hline
\end{tabular}

Tabela 6 Valores absolutos de gravidade ajustados, suas correções e seus respectivos desvios padrão.

\begin{tabular}{c|c|c|c}
\hline $\begin{array}{c}\text { Código da } \\
\text { estação }\end{array}$ & $\begin{array}{c}\text { Correções } \\
(\mu \mathrm{Gal})\end{array}$ & $\begin{array}{c}\text { Valores de g } \\
(\mu \mathrm{Gal})\end{array}$ & $\begin{array}{c}\sigma \\
(\mu \mathrm{Gal})\end{array}$ \\
\hline \hline 016080 & $-2,5$ & 978791662 & 7 \\
\hline CAL02 & $-2,9$ & 978601075 & 5 \\
\hline CAL03 & 12,0 & 978419495 & 6 \\
\hline CAL04 & 8,6 & 978325523 & 6 \\
\hline CAL05 & $-10,4$ & 978162990 & 7 \\
\hline \hline
\end{tabular}

O maior valor residual para as leituras instrumentais foi de $6.5 .10^{-3} \mathrm{UI}$. Os valores desses resíduos ficaram abaixo do desvio padrão estimado inicialmente para as leituras instrumentais (Fig.5). Os valores dos resíduos ficaram na ordem do microgal devido suas leituras possuírem resolução de $1 \mu \mathrm{Gal}$ comprovando o que foi dito no início do trabalho.

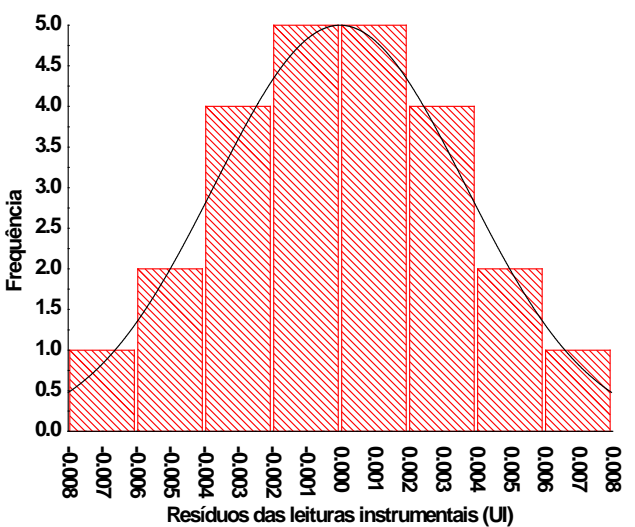

Figura 5 Histograma dos resíduos das leituras instrumentais. Em preto, uma curva Gaussiana com o mesmo valor médio e desvio padrão $(0,000 \pm 0,004 \mathrm{UI})$.

O maior resíduo para os coeficientes das funções de calibração foi de $-0,6.10^{-6} \mathrm{mGal} / \mathrm{Ul}$, que é menor comparado com o desvio padrão estimado inicialmente para essas grandezas observáveis, que são indicadores do excelente comportamento instrumental na LCONAN (Fig.6). Tendo em vista que os efeitos de temperatura e pressão não influenciaram na qualidade dos dados dos CG5, mostrando que os equipamentos estão em perfeitas condições de funcionamento.
Os fatores lineares de escala dos gravímetros CG5 foram tratados como parâmetros no ajustamento e suas precisões ficaram $5.10^{-5}$ (Tab.7).

Tabela 7 Os valores dos fatores lineares de escala ajustados e seus respectivos desvios padrão.

\begin{tabular}{c|c|c}
\hline \hline Gravímetro & $\begin{array}{c}\text { Fatores lineares de } \\
\text { escala }\end{array}$ & $\sigma$ \\
\hline \hline 40343 & 1,000762 & 0,000050 \\
\hline 40347 & 1,000418 & 0,000050 \\
\hline 40600 & 1,000001 & 0,000050 \\
\hline \hline
\end{tabular}
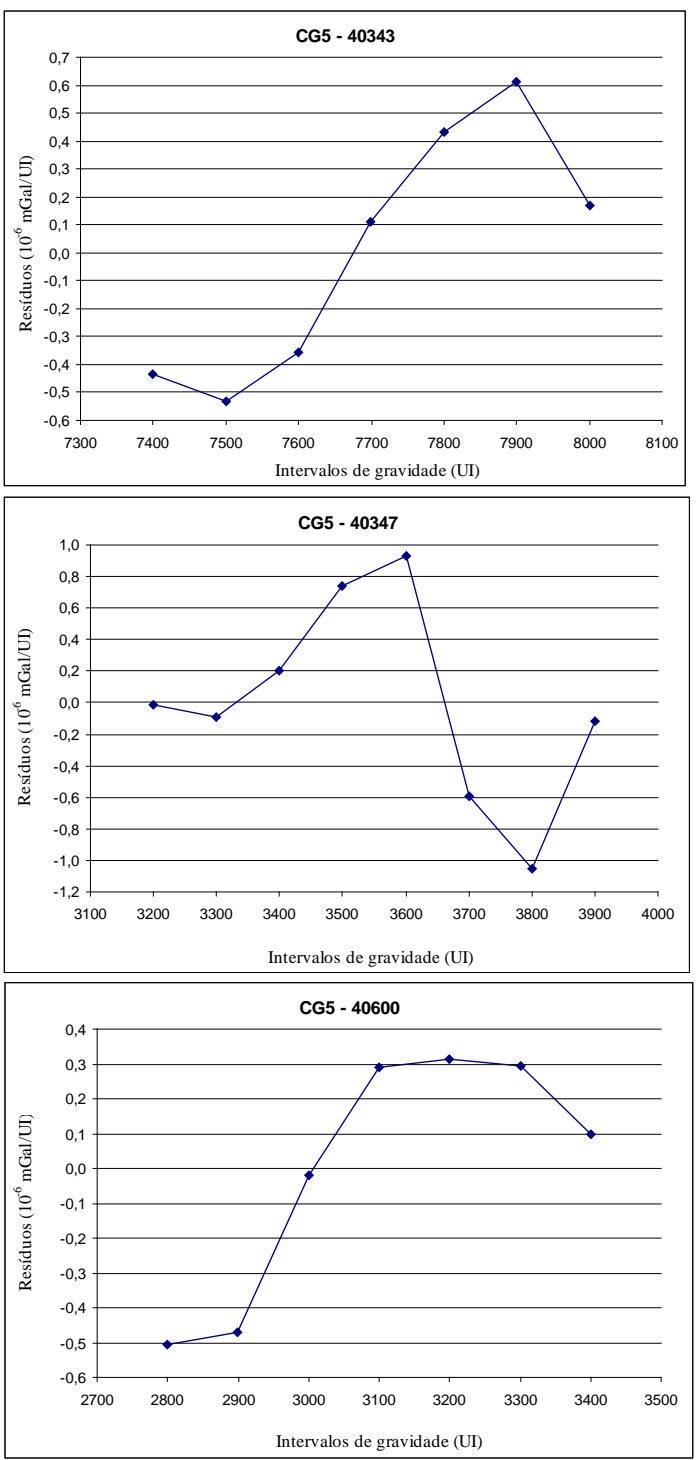

Figura 6 A distribuição dos resíduos dos coeficientes da função de calibração.

\section{Conclusões}

A aferição dos gravímetros CG5 em relação à variação da altitude mostra bem coerente com as suas 
especificações e teve um comportamento satisfatório quando submetido a variações bruscas de temperatura e pressão, como é o caso da LCONAN. A LCONAN fornece aproximadamente a metade da amplitude de gravidade do Brasil. Como a gravidade varia também em latitude, deve-se desenvolver um estudo posterior desde o Rio de Janeiro até a estação de Rivera (URU), para verificar a comportamento desses gravímetros com a variação da latitude.

Os resíduos das leituras instrumentais na ordem do microgal mostraram a qualidade das observações que esses gravímetros digitais CG5 podem medir. Agora, para amplitudes maiores de gravidade deve-se verificar se apenas um fator linear de escala é suficiente (Berquó et al, 2011).

A criação de uma tabela genérica para o gravímetro CG5 facilita a verificação do comportamento instrumental ao ser submetido a um ensaio de calibração. Os resultados apresentados para os coeficientes das funções de calibração também mostraram que os CG5 estão funcionando em perfeitas condições, tendo resíduos abaixo da precisão usada inicialmente no ajustamento.

Qualquer ineficácia refletirá no desempenho desses CG5, como também nas suas características, que estão sujeitas as variações, podendo comprometer a qualidade das medidas. Escala e deriva são características muito afetadas por esse tipo de problema. Assim, com reocupações periódicas da LCONAN será possível detectar eventuais mudanças nessas características, que podem estar associadas ao mau funcionamento, por exemplo, aos dispositivos térmicos e barométricos.

\section{6 - REFERÊNCIAS BIBLIOGRÁFICAS}

BERQUÓ, F.R., ESCOBAR, I.P., PAPA, A.R.R. "Ajustamento de observações gravimétricas com precisão de microgal". Congresso Internacional da Sociedade Brasileira de Geofísica, 2011.

DIAS, F.J.S.S., ESCOBAR, I.P., "A model for adjustment of differential gravity measurements with simultaneous gravimeter calibration", Journal of Geodesy 75: 151156, 2001.

ESCOBAR, I.P. "Injunções relativas em ajustamento gravimétrico". Publicação do Observatório Nacional, dissertação de mestrado, UFPR, Curitiba, PR, Brasil, No.2, 1986.

ESCOBAR, I.P. "Rede gravimétrica fundamental brasileira, uma tradição de 10 anos no Observatório Nacional", Revista Geofísica, No. 26, janeiro-junho, 1987.

ESCOBAR, I.P, SÁ, N.C., DANTAS, J.J., DIAS, F.J.S.S. "The Observatório Nacional Agulhas Negras gravity calibration line". Brazilian Journal of Geophysics, volume 14, número 1, 1996.

FAGGION, P.L, DE FREITAS, S.R.C., GEMAEL, C., SIMÕES, K., JUNIOR, J.S.S., SANTOS JUNIOR, G. "Controle da Função de Transferência de Gravímetros a mola: Metodologia e resultados obtidos na UFPR".
Anais do Simpósio Brasileiro de Geomática, Presidente Prudente, SP, 9-13 de Julho de 2002, p.34-41.

FREITAS, S.R.C. "Marés Gravimétricas: Implicações para a Placa Sul-Americana". Tese de Doutorado, Departamento de Geofísica IAG/USP, São Paulo, 1993.

GEMAEL, C. "Introdução ao ajustamento de observações. Aplicações geodésicas", Ed. Universidade Federal do Paraná, 319 pp., 1994.

LONGMAN, I.M.. "Formulas for computing the tide acceleration due to the Moon and the Sun". J. Geophys. Res. 64: 2351-2356, 1959.

OJA, T., TÜRK, K., ELLMANN, A. "Calibration results of different type spring gravimeters from the repeated measurements of Estonian calibration line". NKG General Assembly, 2010.

SANTOS JÚNIOR, G., FREITAS, S.R.C., GEMAEL, C., FAGGION, P.L. "Implicações em ajustar uma rede gravimétrica com observações médias ou independentes: análise de precisão e confiabilidade". Revista Brasileira de Cartografia, N57/02, 2005.

SCINTREX. CG-5 Scintrex Autograv System Operation Manual, Revision 7, Canada, 2010.

SOUSA, M.A, SANTOS, A.A. "Absolute Gravimetry on the Agulhas Negras Calibration Line". Revista Brasileira de Geofísica, Vol.28(2), 2010.

YUSHKIN, V.D. "Operating Experience with CG5 gravimeters". Measurement Techniques, Vol. 54, No. 5, August, 2011.

\section{Agradecimentos}

Os autores expressam seus agradecimentos ao Departamento de Geofísica do Observatório Nacional, pelo apoio institucional que tornou possível a realização deste trabalho. À CAPES pela bolsa de Doutorado de Francismar Rimoli Berquó. Ao Cnpq pela bolsa de produtividade de Andrés R. R. Papa. 